\title{
Modeling of atrophy size trajectories: variable transformation, prediction and age-of-onset estimation
}

\author{
Charlotte Behning ${ }^{1 *}$, Monika Fleckenstein ${ }^{2}$, Maximilian Pfau ${ }^{3}$, Christine Adrion $^{4}$, Lukas Goerdt ${ }^{5}$, \\ Moritz Lindner ${ }^{5}$, Steffen Schmitz-Valckenberg ${ }^{2}$, Frank G Holz ${ }^{5}$ and Matthias Schmid ${ }^{1}$
}

\begin{abstract}
Background: To model the progression of geographic atrophy $(G A)$ in patients with age-related macular degeneration (AMD) by building a suitable statistical regression model for GA size measurements obtained from fundus autofluorescence imaging.

Methods: Based on theoretical considerations, we develop a linear mixed-effects model for GA size progression that incorporates covariable-dependent enlargement rates as well as correlations between longitudinally collected GA size measurements. To capture nonlinear progression in a flexible way, we systematically assess Box-Cox transformations with different transformation parameters $\lambda$. Model evaluation is performed on data collected for two longitudinal, prospective multi-center cohort studies on GA size progression.

Results: A transformation parameter of $\lambda=0.45$ yielded the best model fit regarding the Akaike information criterion (AIC). When hypertension and hypercholesterolemia were included as risk factors in the model, they showed an association with progression of GA size. The mean estimated age-of-onset in this model was $67.21 \pm 6.49$ years.

Conclusions: We provide a comprehensive framework for modeling the course of uni- or bilateral GA size progression in longitudinal observational studies. Specifically, the model allows for age-of-onset estimation, identification of risk factors and prediction of future GA size. A square-root transformation of atrophy size is recommended before model fitting.
\end{abstract}

Keywords: Geographic atrophy, Age-related macular degeneration, Box-Cox transformation, Mixed-effects models, Prediction, Age-of-onset estimation

\section{Background}

Age-related macular degeneration (AMD) is a leading cause of blindness, especially for people in developed countries older than 60 years $[1,2]$. AMD has two late stages: choroidal neovascularization $(\mathrm{CNV})$ and geographic atrophy (GA). Here we consider GA, which is thought to be the end stage of AMD when CNV does

\footnotetext{
*Correspondence: charlotte.behning@imbie.uni-bonn.de

'Department of Medical Biometry, Informatics and Epidemiology, University

Hospital Bonn, Venusberg-Campus 1, 53127 Bonn, Germany

Full list of author information is available at the end of the article
}

not develop [3] and which is responsible for vision loss in approximately $20 \%$ of all patients with AMD [4]. More than five million people are estimated to be affected by GA worldwide, a number which is supposed to increase with the aging of the population [2]. To date, there is no effective standard treatment available [5].

GA is defined by atrophic lesions of the outer retina resulting from loss of retinal pigment epithelium (RPE), photoreceptors and underlying choriocapillaris (reviewed by [6]). These areas enlarge with time and lead to irreversible loss of visual function [7]. A relevant clinical measure 
of disease progression is the eye-specific size of GA which can be quantified based on imaging techniques including color fundus photography, spectral domain optical coherence tomography imaging, or fundus autofluorescence (FAF) imaging $[8,9]$.

A better understanding of the risk factors that accelerate GA size progression is necessary for the development of treatment options, in particular for the design of (interventional) clinical trials. To date, empirical evidence on GA size progression is usually collected through longitudinal observational studies (e.g. [10-12]). In these studies, it is essential to analyze GA size trajectories over time using an adequate statistical model. Specifically, in the absence of a randomized study design, data analysis needs to account for confounding issues as well as correlation patterns, for instance when both eyes of a patient are included in the study. In the latter case, the correlations between the eyes within one patient need to be incorporated as well as the correlations due to repeated measurements over time.

The aim of this analysis is to systematically derive a statistical approach for modeling GA size in observational ophthalmologic studies. As will be demonstrated in the following sections, the proposed approach generalizes various statistical models for GA size progression that have been used in previous publications (see below). Special focus will be given to the following issues, which are considered to be of particular importance for the planning and design of future interventional trials:

(i) Transformation of GA size. Before model fitting, it is important to consider whether the response (here, GA size) should be transformed. Finding an appropriate transformation can provide information about the underlying natural processes that drive the progression of GA. In recent publications on GA size progression, there has been an ongoing discussion about the optimal choice of transformation [11, 13-15]. Three main modeling paradigms have emerged: The first set of models assumes a linear relationship between GA size and covariables (e.g. risk factors or confounding variables). This implies a constant enlargement of GA size over time. Examples of this modeling approach can be found in [13, 14]. The second approach assumes a quadratic enlargement of the lesion size. This is motivated by the thought of circular atrophic lesions that constantly enlarge with their radiuses $[11,15]$. The third model type is an exponential model in which atrophic lesions enlarge exponentially. Compared to a linear growth model, Dreyhaupt et al. [13] found that the assumption of exponential growth led to improved model fits.

(ii) Age-of-onset estimation. Another relevant topic for modeling GA size progression is the estimation of the age of disease onset. Research on this topic is motivated by the fact that in many clinical trials patients can only be included when the disease is already manifested in a later stage. The estimated age-of-onset may, in contrast to lesion size, be considered as time-invariant variable, and facilitate association analyses with other time-invariant variables such as the genotype.

(iii) Identification of risk factors and confounding variables. For the development of AMD treatments, it is essential to specify meaningful inclusion and exclusion criteria for use in future clinical trials. It is therefore of high importance to identify relevant risk factors and confounding variables, and to analyze their relationships with GA size progression. Such an analysis can be achieved by building a multivariable regression model from observational data that includes relevant risk factors and confounders as covariables.

To address the issues described above, we derive a statistical regression model that includes (possibly transformed versions of) GA size as response variable, as well as potential risk factors and/or confounders (such as e.g. age, smoking) as covariables. To account for the above mentioned correlations between eyes of the same patient as well as temporal correlations, we investigate the use of a mixed-effects modeling approach with patient- and eye-specific random effects terms. In this framework, we identify the "optimal" transformation of GA size by conducting a systematic search within the family of Box-Cox transformations [16]. As will be shown, this systematic approach also allows for the derivation of formulas for age-of-onset estimation. Furthermore, we demonstrate how predictions of future (untransformed) GA size values can be obtained from the fitted regression model.

For model derivation and illustration, we will apply the proposed methods to a data set collected by the multicenter Fundus Autofluorescence in AMD (FAM) study (NCT00393692) and by its single-center extension study, the Directional Spread in Geographic Atrophy (DSGA) study (NCT02051998). These noninterventional, prospective natural history studies adhered to the tenets of the Declaration of Helsinki and were approved by the institutional review boards of the participating centers. Written informed consent was obtained from each participant after explanation of the studies' nature and possible consequences of participation.

\section{Methods}

Data

The data set used here was collected from patients with GA secondary to AMD that were recruited for the FAM study and followed-up in the DSGA study. 
The inclusion and exclusion criteria have been described elsewhere [14, 17]. In brief, the two studies included eyes without any history of retinal surgery, radiation therapy, laser photocoagulation or retinal diseases other than AMD. GA size measurements were obtained by grading FAF retinal images that were recorded at the baseline and follow-up visits. Data was only used for statistical analysis if the difference in total GA size between two graders was smaller than $0.15 \mathrm{~mm}^{2}$ and if the patients had at least two visits.

Our analysis data set contained $N=150$ eyes from $n=101$ patients that where examined in up to nine follow-up visits. At baseline, the median age was 75.7 years (IQR: 70.7 - 80.6 years); $61.4 \%$ of the patients were female, and the mean follow-up time was 3.36 years (range $0.5-13.7$ years) due to the extension by the second study. The GA size varied strongly between eyes: mean GA size at baseline was $5.64 \mathrm{~mm}^{2}$, ranging between $0.07 \mathrm{~mm}^{2}$ and $31.41 \mathrm{~mm}^{2}$. The status of hypertension and hypercholesterolemia was assessed by a patient-reported questionnaire at the baseline visit. Information was obtained based on patients' reports and current medication; medical reports were included in the assessment if available. For details see Table 1.

\section{Regression modeling}

Within a typical ophthalmologic study setting, patients participate in several follow-up visits at which one or both eyes are examined. This leads to correlated measurements, both within the patients and over time. Thus, a model is needed that captures complex correlation structures. A popular regression model, which has been used regularly in the literature on GA $[11,13]$ and which is also considered here, is a mixed-effects model with random effects terms for both eye and patient. Yet, there exists a variety of model specifications and the specific structure is still a matter of debate [18].

Before introducing the full mixed-effects model with possible risk factors and confounders, we start with a model that contains a time trend as only (continuous) covariable. This model serves as a basic model that captures the time dependency of GA enlargement.

Mixed-effects model with time as only covariable. As suggested by Shen et al. [18], we follow the hypothesis that the progression of GA has an underlying process of GA expansion that is mostly the same over time for all eyes. Differences in eyes may arise due to different exposition to environmental conditions, and, most importantly, GA size varies between patients as they enter the study at different time points in their disease history. We therefore propose to include the disease age $\Delta_{i} \geq 0$ of an eye $i$ at study entry directly in the model. We further assume that the atrophy size $y_{i t}$ of an eye $i$ depends on the (unknown) age of the
Table 1 Characteristics of the analysis data set used for statistical modeling

\begin{tabular}{|c|c|c|}
\hline & Count & Percent \\
\hline Patients (n) & 101 & \\
\hline Eyes (N) & 150 & \\
\hline Bilateral GA & 49 & $48.50^{\circ}$ \\
\hline Unilateral GA & 52 & $51.50 \%$ \\
\hline \multicolumn{3}{|l|}{ Hypertension } \\
\hline yes & 56 & $55.40 \%$ \\
\hline no & 44 & 38.60 \\
\hline \multicolumn{3}{|c|}{ Hypercholesterolemia } \\
\hline yes & 28 & 27.70 \\
\hline no & 70 & 69.30 \\
\hline \multicolumn{3}{|c|}{ No. of patients with no. of visits } \\
\hline 2 visits & 25 & 24.75 \\
\hline 3 visits & 23 & 22.78 \\
\hline 4 visits & 23 & 22.78 \\
\hline \multirow[t]{2}{*}{ 5-9 visits } & 30 & 29.70 \\
\hline & Mean (Range) & Median (IQ \\
\hline Age at baseline & 75.61 & 75. \\
\hline [years] & $(57.23-95.06)$ & $(70.67-80.6$ \\
\hline Follow-up time & 3.36 & \\
\hline [years] & $(0.50-13.70)$ & $(1.61-4.5$ \\
\hline GA size at baseline & 5.64 & \\
\hline $\mathrm{mm}^{2}$ & $(0.07-31.40)$ & $(1.76-7.6$ \\
\hline
\end{tabular}

All data considered in this paper was collected from patients with GA secondary to AMD that were recruited for the FAM study. If further monitoring of these patients was performed via the DSGA study, the further progression is included in the analysis data set

disease at study entry $\Delta_{i}$ and the (observable) follow-up time $t \geq 0$ that has passed since. Time is assumed to be measured on a continuous scale, e.g. in days or years since baseline. Under the assumptions by Shen et al. [18], and considering (for the moment) a linear enlargement of GA, this leads to the following regression model:

$$
y_{i t}=\beta \cdot\left(\Delta_{i}+t\right)+\epsilon_{i t},
$$

where $\beta$ denotes the regression slope (i.e. the constant enlargement rate). The residuals $\epsilon_{i t}, i=1, \ldots, N$, are assumed to be normally distributed with zero mean and variance $\sigma^{2}$.

If it is further assumed that the disease age at study entry can be approximated by a normal distribution, the model in (1) can be parameterized such that it becomes a linear mixed-effects model. This is seen by defining $\theta_{i}:=\beta \cdot \Delta_{i} \sim$ $\mathcal{N}\left(\mu_{\theta}, \sigma_{\theta}^{2}\right)$ and $\alpha_{i}:=\theta_{i}-\mu_{\theta} \sim \mathcal{N}\left(0, \sigma_{\theta}^{2}\right)$, so that Model (1) can be written as

$$
y_{i t}=\mu_{\theta}+\beta t+\alpha_{i}+\epsilon_{i t} .
$$

In this form, the model reads as follows: The atrophy size $y_{i t}$ depends on a fixed intercept $\mu_{\theta}$, an eye-specific random intercept $\alpha_{i}$ that reflects the deviation of the disease 
age of eye $i$ at study entry from the mean disease age at study entry, and an overall linear time trend $\beta t$ that is the same for all eyes.

When there are patients in the study that contributed data from both eyes, one needs to consider the nested data structure and account for the correlations between measurements taken from the same patient. This can be done by extending the model equation as follows:

$$
y_{i j t}=\mu_{\theta}+\beta t+\zeta_{j}+\alpha_{i}+\epsilon_{i j t}
$$

where $\zeta_{j} \sim \mathcal{N}\left(0, \sigma_{\zeta}^{2}\right), j=1, \ldots, n$, is a normally distributed patient effect and $\alpha_{i}$ the effect of an 'eye within a patient.' Note: While it is assumed that the residual terms $\epsilon_{i j t}$ are independent of the random effects $\alpha_{i}$ and $\zeta_{j}$, the latter two terms are generally allowed to be correlated. For simplicity, and without loss of generality, we will assume independence of all random effects terms in the following.

Mixed-effects model with covariables. When introducing covariables into the model, it is reasonable to assume that risk factors and/or confounders equally influence the enlargement of GA before and after inclusion of an eye in the study. This assumption can be incorporated in Model (1) by adding a covariable-dependent slope to the model equation:

$$
y_{i t}=\left(\beta+\boldsymbol{\beta}_{x}^{\top} \boldsymbol{x}_{i}\right) \cdot\left(\Delta_{i}+t\right)+\epsilon_{i t},
$$

where $\boldsymbol{x}_{i}=\left(x_{1}, \ldots, x_{k}\right)_{i}^{\top}$ is a vector of $k$ (possibly time-dependent) risk factors for each eye and $\boldsymbol{\beta}_{x}=$ $\left(\beta_{x_{1}}, \ldots, \beta_{x_{k}}\right)^{\top}$ is a vector of parameters that accelerate or slow down GA size progression $\left(\beta_{x_{s}}>0\right.$ and $\beta_{x_{s}}<0$, respectively, $s \in\{1, \ldots k\})$. Note that in the following, we will not distinguish between risk factors and confounders any more, as we assume that both are collected in the vectors $\boldsymbol{x}_{i}$.
Similar to the reparametrization used above, we write $\Delta_{i}:=\left(\mu_{\Delta}+\gamma_{i}\right) \sim \mathcal{N}\left(\mu_{\Delta}, \sigma_{\Delta}^{2}\right)$, where $\mu_{\Delta}$ and $\sigma_{\Delta}^{2}$ denote the mean and the variance of the $i$-the eye at study entry.

The mixed-effects model with covariables can then be written as

$$
\begin{aligned}
y_{i t} & =\left(\beta+\boldsymbol{\beta}_{x}^{\top} \boldsymbol{x}_{i}\right) \mu_{\Delta}+\left(\beta+\boldsymbol{\beta}_{x}^{\top} \boldsymbol{x}_{i}\right) \gamma_{i}+\beta t+\boldsymbol{\beta}_{x}^{\top} \boldsymbol{x}_{i} t+\epsilon_{i t} \\
& =\beta \mu_{\Delta}+\beta t+\mu_{\Delta} \boldsymbol{\beta}_{x}^{\top} \boldsymbol{x}_{i}+\boldsymbol{\beta}_{x}^{\top} \boldsymbol{x}_{i} t+\beta \gamma_{i}+\boldsymbol{\beta}_{x}^{\top} \boldsymbol{x}_{i} \gamma_{i}+\epsilon_{i t} .
\end{aligned}
$$

with eye-specific random effects $\gamma_{i} \sim \mathcal{N}\left(0, \sigma_{\Delta}^{2}\right)$. The linear enlargement in Model (5) thus implies dependency of $y_{i t}$ on an interaction term between $t$ and $\boldsymbol{x}_{i}$, and also on random slopes of the covariable values $\boldsymbol{x}_{i}$. Importantly, Eq. 5 implies numerous dependencies between the slope parameters associated with $t, \boldsymbol{x}_{i}, \boldsymbol{x}_{i} t, \gamma_{i}$, and $\boldsymbol{x}_{i} \gamma_{i}$, so that the model no longer possesses the structure of a "standard" mixed-effects model with unrestricted estimation of coefficients. Details on model fitting will be given below.

Finally, when considering patients that contribute data from both eyes, one specifies

$$
\begin{aligned}
y_{i j t} & =\beta \mu_{\Delta}+\beta t+\mu_{\Delta} \boldsymbol{\beta}_{x}^{\top} \boldsymbol{x}_{i}+\boldsymbol{\beta}_{x}^{\top} \boldsymbol{x}_{i} t+\beta \gamma_{i} \\
& +\boldsymbol{\beta}_{x}^{\top} \boldsymbol{x}_{i} \gamma_{i}+\beta \zeta_{j}+\boldsymbol{\beta}_{x}^{\top} \boldsymbol{x}_{i} \zeta_{j}+\epsilon_{i j t}
\end{aligned}
$$

with patient-specific random effects $\zeta_{j} \sim \mathcal{N}\left(0, \sigma_{\zeta}^{2}\right), j=$ $1, \ldots, n$, and an additional interaction term between $\boldsymbol{x}_{i}$ and $\zeta_{j}$.

The model equations presented so far ascribe a linear relationship between time, risk factors, and GA size. In the following section, possible transformations are examined, so that the modeling approach is extended to model nonlinear progressions.

\section{Transformation of the response}

As an example, Fig. 1 A shows the GA size trajectories of four eyes contained in the analysis data set. Considering

\section{A}



B

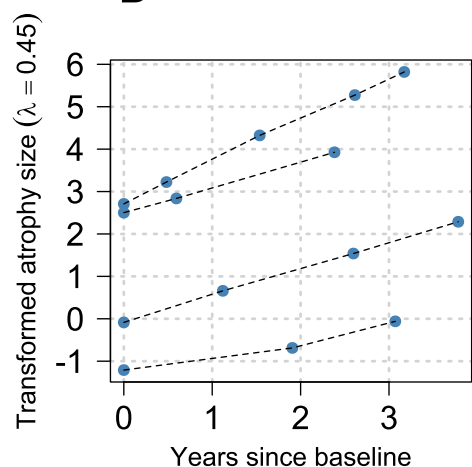

Fig. 1 Progression of GA size. A Untransformed GA size trajectories of four different eyes contained in the analysis data set and $\mathbf{B}$ trajectories on a transformed scale with transformation parameter $\lambda=0.45$ 
these progressions, it is conceivable to assume that the trajectories are not strictly linear. Since the model equations above (Models (1) to (6)) refer to linear enlargement processes, a transformation of the response is convenient for modeling non-linear progression (see Fig. 1B).

Three different transformation approaches have been used in recent publications on GA size progression (e.g. [11, 13-15]): (i) Linear models with no response transformation implying a linear relationship between GA size and the covariables, (ii) linear models with square root transformation of the response, and (iii) linear models with log-transformed response - or equivalently exponentially transformed models with no transformed response - implying an exponential enlargement of the lesion size.

Box-Cox transformation Instead of comparing only the most commonly used transformations, we consider a systematic and more comprehensive strategy for finding an appropriate transformation of the GA size. For this systematic approach, the Box-Cox model class is applied because it covers a wide range of transformations, including the transformations (i) to (iii) above. More specifically, for an atrophy size $y>0$ we consider the class of Box-Cox transformations

$$
f_{\lambda}(y):=y^{(\lambda)}= \begin{cases}\frac{y^{\lambda}-1}{\lambda} & \text { if } \lambda \neq 0 \\ \log (y) & \text { if } \lambda=0\end{cases}
$$

as introduced by [16]. Applying (7) to one of the Models (1)-(6) reads as follows: $\lambda=1$ refers to a model with no response transformation, $\lambda=0.5$ corresponds to a square-root transformation of the response and $\lambda=0$ can be interpreted as exponential enlargement of the GA size.

Model comparison The main criterion used for our model comparisons was Akaike's Information Criterion (AIC) [19]. More specifically, our aim was to choose the transformation parameter $\lambda$ that minimized AIC on the analysis data set while assuring that the assumptions of Models (1) to (6) were best possibly met, in particular the normality of the residuals. The AIC is defined by AIC $=-2 \cdot \log (L)+2 \cdot n_{\text {params }}$, where $L$ is the likelihood of the model under consideration (evaluated at the maximum likelihood estimate) and $n_{\text {params }}$ denotes the number of parameters used in the model. As we compared models with a transformed response, we applied the density transformation theorem to compute the likelihood $L$.

Maximum likelihood estimation The estimation of the model parameters was performed by maximum likelihood (ML) estimation. ML estimation was carried out for a grid of fixed transformation parameters $\lambda$ using the transformed GA size values. Subsequently, the likelihoods were compared and the transformation parameter referring to the model with minimum AIC was considered best.

We initially assumed that there was an "optimal" value $\lambda$ for which the transformed atrophy size given the random effects followed a normal distribution. In addition, we briefly considered random effects with an unspecified mixing distribution as a non-parametric cross-check. The two approaches will be described in the next paragraphs.

Normally distributed random effects As noted above, the linear model in (6) imposes numerous side conditions on the slope parameters associated with $t, \boldsymbol{x}_{i}, \boldsymbol{x}_{i} t, \gamma_{i}$, and $\boldsymbol{x}_{i} \gamma_{i}$. In order to fit Model (6) using readily available software for the estimation of the slope parameters (without side conditions, such as the R add-on package lme4[20], version 1.1-25), we propose to iterate the following steps:

(i) For given estimates $\hat{\beta}$ and $\hat{\boldsymbol{\beta}}_{x}$ compute the values of the working covariable $\tilde{x}_{i}:=\hat{\beta}+\hat{\boldsymbol{\beta}}_{x}^{\top} \boldsymbol{x}_{i}$.

(ii) Fit the linear mixed-effects model

$$
y_{i j t}=\beta t+\boldsymbol{\beta}_{x}^{\top} \boldsymbol{x}_{i} t+\mu_{\Delta} \tilde{x}_{i}+\tilde{x}_{i} \gamma_{i}+\tilde{x}_{i} \zeta_{j}+\epsilon_{i j t}
$$

to obtain updates of the coefficient estimates of $\hat{\mu}_{\Delta}$, $\hat{\beta}$, and $\hat{\boldsymbol{\beta}}_{x}$. Note, that Model (8) is just a re-formulation of Model (6) that can be fitted without side conditions on its slope parameters. For the fitting procedure a fixed intercept term is added to increase computational stability and to relax the condition that the empirical mean of estimated random effects terms is forced to be zero.

The starting values for $\hat{\beta}$ and $\hat{\boldsymbol{\beta}}_{x}$ in Step (i) may be obtained from (8) with an initial value of $\tilde{x}_{i}=1$. As demonstrated in the supplementary materials (see Additional file 1), repeated execution of (i) and (ii) will typically converge to the final estimates after less than 20 iterations.

Random effects with unspecified mixing distribution As an alternative to mixed-effects modeling with normally distributed terms, Almohaimeed et al. [21] proposed to consider a nonparametric maximum likelihood (NPML) approach. This approach approximates the distribution of each random effect by a discrete distribution with finite number of mass points $K$. It then uses an expectationmaximization algorithm to find the nonparametric maximum likelihood estimate. Here, the NPML approach is used to verify the optimal transformation parameter obtained from modeling with normally distributed random effects.

\section{Age-of-onset estimation}

Model without covariables As defined by [22], a diagnosis for GA can be given at a minimum lesion diameter of $250 \mu \mathrm{m}$ and thus a lesion area of $0.05 \mathrm{~mm}^{2}$. Based on 
this specification and denoting $\lambda_{\text {opt }}$ as the value of $\lambda$ that is optimal w.r.t. AIC, the time $\hat{t}_{0_{i j}}$ at which the atrophy size was $\hat{y}_{i j t_{0}}=0.05\left[\mathrm{~mm}^{2}\right]$ (i.e. $\hat{y}_{i j t_{0}}^{(\lambda)}=\lambda_{\text {opt }}^{-1} \cdot\left(0.05^{\lambda_{\text {opt }}}-1\right)$ ) can be obtained by solving the model equation of the transformed mixed-effects Model (3) for $t$ :

$$
\hat{t}_{0_{i j}}=\frac{\lambda_{\text {opt }}^{-1} \cdot\left(0.05^{\lambda_{\text {opt }}}-1\right)-\left(\hat{\mu}_{\theta}+\hat{\zeta}_{j}+\hat{\alpha}_{i}\right)}{\hat{\beta}},
$$

where $\hat{\beta}$ and $\hat{\mu}_{\theta}$ denote the ML estimates of $\beta$ and $\mu_{\theta}$, respectively, and $\hat{\zeta}_{j}$ and $\hat{\alpha}_{i}$ denote the realizations of the random effect terms. As a consequence, subtracting the estimated time $\hat{t}_{0_{i j}}$ from the patient's age at study entry results in the estimated age-of-onset of GA in the $i$-th eye of patient $j$. Remark: While from a modeling perspective a theoretical atrophy size of $y_{i j t_{0}}=0 \mathrm{~mm}^{2}$ could be defined at the time of disease onset, we will focus on the clinically relevant definition $\left(y_{i j t_{0}}=0.05 \mathrm{~mm}^{2}\right)$ here. For $y=0$ it holds that $t_{0_{i j}}=\Delta_{i j}=\frac{1}{\beta} \cdot\left(\mu_{\theta}+\zeta_{j}+\alpha_{i}\right)$.

Model with covariables Analogous to (9) one can estimate the ages of GA onset of the study eyes in a model with additional covariables. From Eq. 8 one obtains

$$
\hat{t}_{0_{i j}}=\frac{\lambda_{o p t}^{-1} \cdot\left(0.05^{\lambda_{o p t}}-1\right)-\tilde{x}_{i}\left(\hat{\mu}_{\Delta}+\hat{\zeta}_{j}+\hat{\alpha}_{i}\right)}{\tilde{x}_{i}}
$$

where $\tilde{x}_{i}:=\hat{\beta}+\hat{\boldsymbol{\beta}}_{x}^{\top} \boldsymbol{x}_{i}$ contains the parameters obtained from ML estimation.

\section{Prediction}

Evaluating a model and its coefficients only on a transformed scale is challenging as the linearity of the predictor-response relationships in Models (5) and (6) only holds on the transformed scale but not on the original scale of the response (provided that $\lambda \neq 1$ ). As a consequence, the calculation of the expected GA size $\mathbb{E}(y \mid x)$
- and hence any prediction of expected disease progression - cannot be done in an unbiased way by a simple back-transformation.

To see this, consider a non-linear Box-Cox transformation $f(y)$ with an arbitrary parameter $\lambda \neq 1$ and, where existent, the corresponding inverse Box-Cox transformation $f^{-1}(y)$. Further, let $f\left(y_{i j t} \mid \boldsymbol{x}_{i}\right)=z_{i j t}+\epsilon_{i j t}$, where $z_{i j t}:=\mathbb{E}\left(f\left(y_{i j t} \mid \boldsymbol{x}_{i}\right)\right)$ and $\epsilon_{i j t}$ denote the linear predictor and the residual, respectively in one of the above models. A naive back-transformation would directly take the inverse of the linear predictor, i.e. $f^{-1}\left(z_{i j t}\right)$, which differs from the desired expected GA size value $\mathbb{E}\left(y_{i j t} \mid \boldsymbol{x}_{i}\right)=$ $\mathbb{E}\left(f^{-1}\left(z_{i j t}+\epsilon_{i j t}\right)\right)$ by Jensens's inequality [23]. In other words, $f^{-1}\left(\mathbb{E}\left(f\left(y_{i j t} \mid \boldsymbol{x}_{i}\right)\right)\right) \neq \mathbb{E}\left(y_{i j t} \mid \boldsymbol{x}_{i}\right)$. To address this issue and to obtain unbiased predictions of the GA size, we propose to sample $r=10,000$ residuals from the empirical distribution $\hat{\epsilon}_{1}, \ldots, \hat{\epsilon}_{r}$ in the respective fitted model. The expected atrophy size on the original scale can

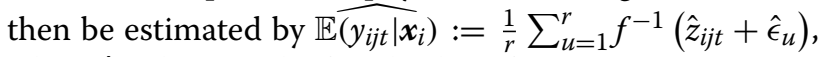
where $\hat{z}_{i j t}$ denotes the fitted value of $f\left(y_{i j t} \mid \boldsymbol{x}_{i}\right)$.

\section{Results}

In this section, we present the results obtained from fitting Models (2), (3) and (6) to the analysis data set (150 eyes of 101 patients). Missing values in the covariables were imputed using the $R$ package mice [24] with one imputation run. Fitting was done using lme4 [20] with the algorithm described above.

\section{Modeling of GA size trajectories}

Determination of the transformation parameter In order to determine the optimal value of the transformation parameter $\lambda$, we evaluated linear mixed-effects models of the forms (3) and (6) on the analysis data set. Box-Cox-transformed responses with varying values of $\lambda$ were considered in each of the models. As seen in Fig. 2A, the minimum AIC value was reached at $\lambda_{\text {opt }}=0.45$ in the model without covariables. The model with covariables
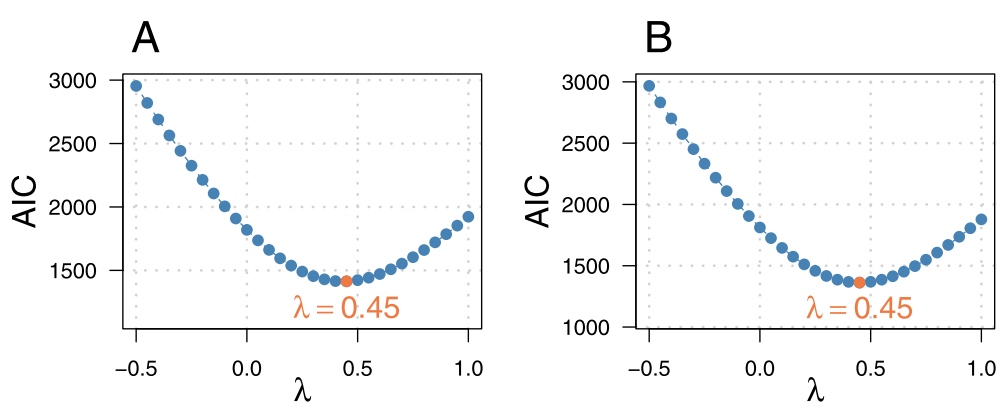

Fig. 2 Determination of the optimal Box-Cox transformation. For each value of the transformation parameter $\lambda$, parametric mixed-effects models $\mathbf{A}$ without covariables as in Model (3) and $\mathbf{B}$ with covariables as in Model (6) were fitted to the analysis data set. Model fitting was performed using the $R$ package Ime4. The orange dot indicates the optimal fit, which was achieved at $\lambda_{\text {opt }}=0.45$. For Model (3), the optimal AIC value was $A I C_{\lambda=0.45}=1413.69$ and for Model (6) the optimal AIC value was $A / C_{\lambda=0.45}=1347.78$ 


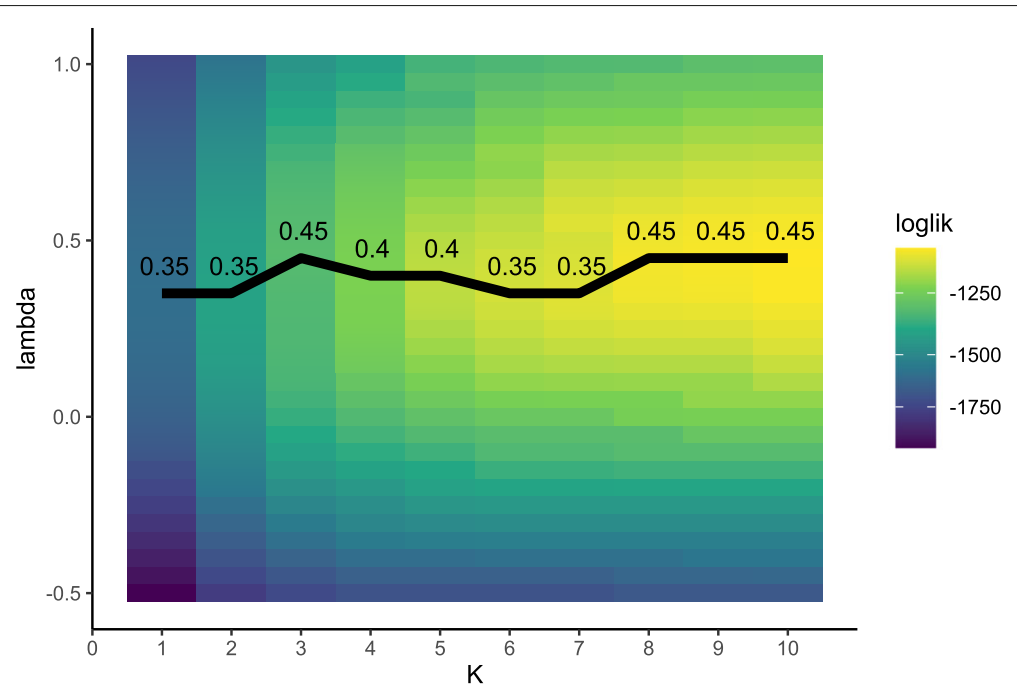

Fig. 3 Optimization using NPML approach. Log-likelihood values obtained from fitting Model (2) to the analysis data set with the NPML method, as implemented in the R package boxcoxmix [21]. The black line indicates the optimal values of the transformation parameter $\lambda$ for varying numbers of mass points $K$. The corresponding values can be found in Table 2

also yielded an optimal AIC value at $\lambda_{\text {opt }}=0.45$ (Fig. 2B).

The NPML approach led to similar results for the optimal value of $\lambda$ in the setting without covariables. As seen in Fig. 3, the obtained values for the optimal $\lambda$ ranged between 0.35 and 0.5 . For a larger number of mass points $(K>7)$ the same optimal $\lambda(=0.45)$ as in the parametric approach was found.

Normality of the residuals Figure 4 shows the residual diagnostics obtained from fitting Model 6 to the analysis data, including hypercholesterolemia and hypertesnsion as risk factors. It is seen that even after transformation the fitted residuals were not normally distributed. However, homoscedasticity was better met after transformation with $\lambda_{\text {opt }}=0.45$. Furthermore, the distribution of the residuals was less skewed after transformation.

Effects of risk factors As shown in Fig. 4, the residuals obtained from fitting Model 6 to the analysis data set did not perfectly follow a normal distribution, even after transformation of the response. Therefore, inference procedures that rely on asymptotic normality may not be the best choice to investigate the effects of risk factors on (transformed) GA size. To address this issue, we used a bootstrap approach to obtain the $95 \%$ confidence intervals of the parameters within Model (6). The results are presented in Table 3 and in Fig. 5. It is seen, that time was associated with the transformed GA size, growing by 0.42 (95\% CI $[0.36,0.50])$ per year. Also the absence of hypercholesterolemia was associated with more rapid enlargement of the lesion size (estimate: $0.11,95 \% \mathrm{CI}[0.06,0.17]$ ), while a slower progression in patients without hypertension (estimate: $-0.09,95 \%$ CI $[-0.17,-0.03]$ ) was found.
Note that the estimated coefficients refer to transformed GA size and thus cannot be directly interpreted in terms of an enlargement of the GA size measured in $\mathrm{mm}^{2}$.

Remark: Model fitting was performed on an imputed data set, using the $R$ package mice [24] with one imputation. Results obtained from complete case analysis were almost identical.

\section{Age-of-onset estimation}

Figure 6 presents the estimated ages of disease onset of the study eyes, as obtained from Models (3) (without covariables) and (6) (with covariables). For the simple model

Table 2 Optimization using NPML approach

\begin{tabular}{llll}
\hline $\mathbf{K}$ & loglik & $\boldsymbol{\lambda}_{\text {opt }}$ & $\mathbf{A I C}_{\boldsymbol{b m}}$ \\
\hline 1.00 & -1593.02 & 0.35 & 3192.04 \\
2.00 & -1402.21 & 0.35 & 2814.41 \\
3.00 & -1310.29 & 0.45 & 2634.57 \\
4.00 & -1230.15 & 0.40 & 2478.30 \\
5.00 & -1164.85 & 0.40 & 2351.70 \\
6.00 & -1142.70 & 0.35 & 2311.39 \\
7.00 & -1127.57 & 0.35 & 2285.14 \\
8.00 & -1107.63 & 0.45 & 2249.26 \\
9.00 & -1102.10 & 0.45 & 2242.19 \\
10.00 & -1096.30 & 0.45 & 2234.60 \\
\hline
\end{tabular}

The table presents the optimal values of the transformation parameter $\lambda$ that were obtained from fitting Model (2) with the boxcoxmix package [21] using the analysis data set. In addition, the respective log-likelihood and AIC bm values (evaluated at the optimal $\lambda$ values) are shown for varying numbers of mass points $K$. Following [21], the information criterion was defined as

$\mathrm{AlC}_{b m}=-2 \log (L)+2 \cdot(p+2 K)$. Hence the AIC values in the fourth column cannot be directly compared to the AIC values presented in Fig. 2 



Fig. 4 Distribution of residuals. Residual diagnostics for Model (6) with transformation parameter $\lambda=1$ (left column) and optimal transformation parameter $\lambda=0.45$ (right column). Note that $\lambda=1$ corresponds to a model with untransformed response. Panels $\mathbf{A}$ and $\mathbf{B}$ present normal quantile-quantile plots of the estimated residuals that were obtained from fitting Model (6) to the analysis data set. Panels $\mathbf{C}$ and $\mathbf{D}$ contain plots of estimated residuals vs. fitted values (fitted values include random effect terms)

without further covariables, the estimated mean age-ofonset was $66.93( \pm 7.56)$ years and for the model with covariables the estimated median age-of-onset was 67.21 $( \pm 6.49)$ years. This is in line with previously reported results, e.g. Li et al. [26] estimated the prevalence of GA in people under 64 years to range between $0.1 \%$ and $0.2 \%$, depending on the country.

\section{Estimation of GA size on the original scale}

To obtain the distribution of GA size on the original scale, we sampled 10,000 times from the empirical distribution of the estimated residuals (obtained from Model (6)) and added these values to the fitted transformed GA size values $f_{\lambda}(y)$ before applying a reverse Box-Cox transformation. The back-transformed expected GA size values are shown in Fig. 7.

The root mean squared difference between the observed GA size and the modeled GA size was $1.10 \mathrm{~mm}^{2}$, implying that estimated expected GA size values deviated by ca. $1 \mathrm{~mm}^{2}$ on average from the true GA size values. The respective mean squared differences for alternative values of the transformation parameter $\lambda$ are shown in Fig. 8 .

Table 3 Analysis of risk factors in the analysis data set

\begin{tabular}{lrrr}
\hline Variable & Estimate & $\mathbf{9 5 \%} \mathbf{C l}$ & $\boldsymbol{p}$-value \\
\hline time [in years] & 0.42 & $(0.36,0.50)$ & $<0.0001$ \\
time $x$ (hyperchol.= no) & 0.11 & $(0.06,0.17)$ & $<0.0001$ \\
time $x$ (hypertension = no) & -0.09 & $(-0.17,-0.03)$ & 0.0004
\end{tabular}

\section{Variance Term}

Eye:Patient $\gamma_{i}$

Patient $\zeta_{j}$

Residuals $\epsilon$
Estimate

$1.83^{2}$

$4.03^{2}$

$0.42^{2}$

The table presents the coefficient estimates and bootstrap 95\% confidence intervals that were obtained from fitting Model (6) with transformed response ( $\lambda=0.45)$ to an imputed version of the analysis data set. The model parameter $\mu_{\Delta}$, which reflects the mean disease age at study entry, was estimated to be $\hat{\mu}_{\Delta}=4.74$ (95\% Cl [3.41, 4.83]). $P$-values were obtained using the R package ImerTest [25] 




Fig. 5 Analysis of risk factors in the analysis data set. The plot shows the coefficient estimates (red dots) that were obtained from fitting Model (6) with transformed response $(\lambda=0.45)$ to an imputed version of the analysis data set. Bootstrap 95\% confidence intervals are indicated by blue lines. For further details see Table 3

As can be seen here, the $\lambda$, that lead to a minimal difference on the original scale, was slightly larger than the optimal $\lambda=0.45$ obtained by AIC-based methods. However, the variation in the average distances between observed and predicted values was rather small (minimal distance $1.05 \mathrm{~mm}^{2}$ at $\lambda=0.55,1.06 \mathrm{~mm}^{2}$ at $\lambda=0.50$, and $1.10 \mathrm{~mm}^{2}$ at $\left.\lambda=0.45\right)$.

Prediction of next observation In clinical context, a prediction of the next observation of a patient already included in a clinical trial might be of interest. For each observed eye, for which values of more than three visits were present, we predicted the last observation. To this purpose we fitted a model to a training data set excluding the last observation while performance was measured on the last observation. The root mean squared difference between observed atrophy sizes and the mean predicted atrophy sizes was $\sqrt{\operatorname{avg}\left((\overline{\hat{y}}-y)^{2}\right)}=1.67 \mathrm{~mm}^{2}$.

\section{Discussion}

Despite a high prevalence and extensive research efforts, there are currently no effective standard treatment



Fig. 6 Age-of-onset estimation. Boxplots of the observed ages at baseline (orange) and the modeled ages of disease onset (Models (3) and (6), blue) of the eyes in the analysis data set



Fig. 7 Agreement between the estimated expected GA size values and the measured GA size values modeled distributions show one boxplot per observation and were generated by sampling from the residual distribution of Model (6), followed by a back-transformation of $z_{i j t}+\epsilon_{u}$ to the original scale. The orange line indicates a perfect fit

options for GA. It is therefore essential to develop accurate models for disease progression that enable researchers to efficiently plan and design clinical trials.

In this article, we presented a comprehensive framework for modeling the course of GA size progression in longitudinal observational studies. Our modeling approach was derived from a linear enlargement model using transformed GA size as response variable. As shown in the Results section, the resulting model can be embedded in the class of linear mixed-effects models [27], allowing for the incorporation of risk factors, confounding variables, and measurements taken repeatedly from the same patients and eyes. Since the assumption of linear enlargement imposes numerous restrictions on the model parameters, it is necessary to adapt standard (unrestricted) mixed-effects modeling approaches to the specific structure of the proposed model. To this purpose, we developed

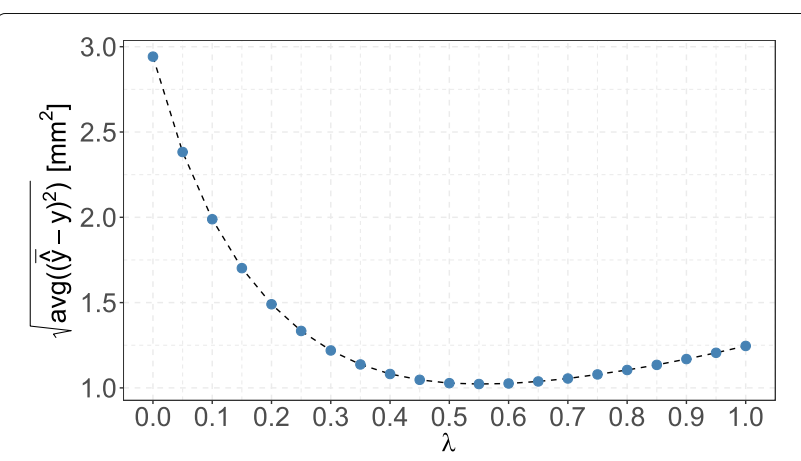

Fig. 8 Root mean squared deviation between modeled response and observed values for different transformation parameters. Deviation was measured by avg $\left((\overline{\hat{y}}-y)^{2}\right)$. Here, the back-transformed estimate was defined by $\hat{y}=f^{-1}\left(\hat{y}^{(\lambda)}+\hat{\epsilon}\right)$ (if $\underline{f}^{-1}$ existed), where $f^{-1}$ was the inverse Box-Cox transformation, and $\overline{\hat{y}}$ was its mean (computed from sampling 10,000 times from the fitted residuals). For $\lambda=0.45$, the deviation was $1.10 \mathrm{~mm}^{2}$ and a minimal squared deviation was reached at $\lambda=0.55$ with a deviation of $1.05 \mathrm{~mm}^{2}$ 
an algorithm for GA size modeling that can be implemented using readily available software for fitting linear mixed-effects models.

To obtain the best transformation of GA size, we conducted a systematic search within the class of Box-Cox transformation models that included both parametric and non-parametric approaches. Our experiments yielded an optimal transformation that was close to the square-root function, thereby justifying earlier modeling strategies that assumed linear trajectories of square-root transformed GA size over time [18]. Of note, the square-root transformation has a straightforward interpretation in terms of a linear enlargement of the atrophy radius [15].

A convenient feature of the proposed modeling approach is that it yields estimates of the disease age of the eyes at study entry. This is important because patients can only be included in trials when the disease has already manifested. When applied to the analysis data set consisting of patients included in the FAM-study, disease age at study entry was estimated to range between 3.5 and 13.4 years (Model (6)). These estimates are in line with estimated prevalence values reported in the literature [4], but the resulting ages of disease onset were smaller than previously modeled ages using data partly from the same study [28].

Since the proposed modeling approach employs a transformed response variable, care has to be taken when making predictions of future values of atrophy size. As argued in the Results section, predictions with a naive back-transformation may show a bias due to the nonlinearity of the square-root function. To address this issue, we proposed a sampling approach that allows for drawing valid conclusions and making undistorted predictions of GA size on its original scale. In the analysis data set, estimated expected GA size values derived from the proposed model deviated $1.10 \mathrm{~mm}^{2}$ on average from the respective observed values.

Generally, the model proposed here allows for performing statistical hypothesis tests on a set of risk factors suspected to accelerate or slow down GA size enlargement. This strategy was illustrated in the Results section, where an analysis of a GA patient sample of the FAM study identified significant interaction effects between hypercholesterolemia, hypertension and time. Although a number of studies have shown a link between cardiovascular risk factors and AMD, the role of hypertension, atherosclerosis, high BMI, diabetes mellitus, higher plasma fibrinogen and hyperlipidaemia remain equivocal owing to inconsistent findings (reviewed in [29]). High blood pressure is shown to be associated with lower choroidal blood flow and disturbed vascular homeostasis [30]. Since perfusion deficits in the choriocapillaris, the innermost layer of the choroid, are associated with future GA progression [31], an asso- ciate between hypertension and increased GA progression appears biologically plausible. Regarding the association of hypercholesterinemia and decreased GA progression, the biological plausibility remains elusive. The majority of previous studies did not find any relationship between systemic cholesterol levels and progression to early AMD, GA or nAMD (reviewed in [29]), although two studies found an association between serum cholesterol on the development of late stage AMD [32, 33]. Interestingly, one of these studies reported that serum cholesterol levels have a protective effect on the development of nAMD, while they are a risk factor for the development of GA [32]. These observations apparently are in contrast to our results; however, there is evidence that different mechanisms may be involved in driving GA enlargement than those increasing the risk of de novo GA development [6]. Further validation of the risk factors, especially on an external data set, is necessary

While it has been established that so-called nascent GA progresses to manifest GA [34], the trajectory of early $\mathrm{GA}$ - prior to the minimum lesion size requirement for clinical trials (e.g., $2.5 \mathrm{~mm}^{2}$ ) - is poorly understood. The information derived by this modeling strategy can be used to design future intervention studies, for example regarding the stratification of patient groups and the definition of inclusion criteria. Of note, the proposed modeling approach is not restricted to established epidemiological covariables like hypertension but may also incorporate novel markers of disease progression such as patientreported outcome measures [35], digital biomarkers, and machine-learning-based scores derived from structural imaging data [36]. The proposed model constitutes a flexible framework to systematically investigate the transition from intermediate to late AMD in large observational studies such as the MACUSTAR study (ClinicalTrials.gov: NCT03349801) [37].

\section{Conclusions}

We have provided a comprehensive framework for modelling the trajectories of uni- or bilateral Ga size progression in longitudinal observational studies. Our analysis shows that a square-root transformation of atropy size is recommended before model fitting. The proposed modelling approach allows for the estimation of age-of-onset, identification of risk factors and prediction of future GA size. The risk factors analyzed here require further validation in an external study population.

\section{Abbreviations}

AIC: Akaike's information criterion; AMD: Age related macular degeneration; avg: Average; BMI: Body max index; Cl: Confidence interval; DSGA: Directional spread in geographic atrophy study; FAF: Fundus autoflourescence; FAM: Fundus autoflourescence in AMD study; GA: Geographic atrophy; IQR: Interquartile range; ML: Maximum likelihood; nAMD: Neovascular AMD; NPML: Nonparametic maximum likelihood; RPE: Retinal pigment epithelium 


\section{Supplementary Information}

The online version contains supplementary material available at https://doi.org/10.1186/s12874-021-01356-0.

\section{Additional file 1: Supplementary information.}

\section{Acknowledgements}

This work was supported by the DFG (German Research Foundation) Grant No FL658/4-1 and FL658/4-2 (MF); PF950/1-1 (MP) Research Priority Program Age-Related Macular Degeneration Grant SPP 1088, Ho 1926/1-3 (FGH); Grants from National Institutes of Health Core Grant (EY014800) (MF); and in part by an Unrestricted Grant from Research to Prevent Blindness, New York, NY, to the Department of Ophthalmology \& Visual Sciences, University of Utah. The authors would like to thank Joanna Czauderna for her support in conducting the DSGA study.

\section{Authors' contributions}

Conception and design: MS, MF, CB. Implementation and analysis: CB. Data collection and interpretation: MF, MP, LG, ML, SSV. Review and approval of final manuscript: CA, MP, MF. All authors read and approved the final manuscript.

\section{Funding}

Open Access funding enabled and organized by Projekt DEAL.

\section{Availability of data and materials}

The datasets generated during and/or analysed during the current study are not publicly available in order to protect the privacy of study participants. However, they are available from the principal investigators of the FAM and DSGA studies on reasonable scientific request.

\section{Declarations}

Ethics approval and consent to participate

This study does not include human subjects. Data presented here are from the Fundus Autofluorescence in AMD (FAM) study (ClinicalTrials.gov Identifier: NCT00393692) and the Directional Spread in Geographic Atrophy (DSGA) study (ClinicalTrials.gov Identifier: NCT02051998). The FAM study was approved by the human ethics committees at the University of Bonn and the local Institutional Review Boards and the local ethics committees at the study centers (University of Bonn, University of Heidelberg, University of Leipzig, Ludwig-Maximilians-University Munich, St. Franziskus Hospital Münster, University of Würzburg). The human ethics committees at the University of Bonn approved the DSGA study. All research adhered to the tenets of the Declaration of Helsinki. All participants provided informed consent.

\section{Consent for publication}

All authors have read and approved the submission of the manuscript.

\section{Competing interests}

Financial disclosures:

S. Schmitz-Valckenberg reports grants from Acucela/Kubota Vision, personal fees from Apellis, grants and personal fees from Novartis, grants and personal fees from Allergan, grants and personal fees from Bayer, grants and personal fees from Bioeg/Formycon, grants, personal fees and non-financial support from Carl Zeiss MediTec AG, grants and non-financial support from Centervue, personal fees from Galimedix, grants, personal fees and non-financial support from Heidelberg Engineering, grants from Katairo, non-financial support from Optos, personal fees from Oxurion, outside the submitted work.

M. Fleckenstein reports grants, personal fees and non-financial support from Heidelberg Engineering, non-financial support from Zeiss Meditech, grants and non-financial support from Optos, personal fees and grant from Novartis, personal fees from Bayer, grants and personal fees from Genentech, from Roche, outside the submitted work; In addition, Dr. Fleckenstein has a patent US20140303013 A1 pending.

F.G. Holz reports grants and personal fees from Acucela, Allergan, Apellis, Bayer, Bioeq/Formycon, Roche/Genentech, Geuder, Heidelberg Engineering, ivericbio, Kanghong, Novartis, Zeiss; personal fees from Boehringer-Ingelheim, Grayburg Vision, LinBioscience, Pixium Vision, Stealth BioTherapeutics, Aerie, Oxurion outside the submitted work.

M. Schmid reports personal fees from Pixium Vision.

The remaining authors have no competing interest.

\section{Author details}

${ }^{1}$ Department of Medical Biometry, Informatics and Epidemiology, University Hospital Bonn, Venusberg-Campus 1, 53127 Bonn, Germany. ${ }^{2}$ John A. Moran Eye Center, University of Utah, Salt Lake City, USA. ${ }^{3}$ Ophthalmic Genetics and Visual Function Branch, National Eye Institute, Bethesda, MD, USA. ${ }^{4}$ Institute for Medical Information Processing, Biometry and Epidemiology, Ludwig-Maximilians-University, Munich, Germany. ${ }^{5}$ Department of Ophthalmology, University Hospital Bonn, Bonn, Germany.

Received: 29 December 2020 Accepted: 22 July 2021

Published online: 17 August 2021

\section{References}

1. Lim LS, Mitchell P, Seddon JM, Holz FG, Wong TY. Age-related macular degeneration. Lancet. 20121728-38.

2. Wong WL, Su X, Li X, Cheung CMG, Klein R, Cheng C-Y, Wong TY. Global prevalence of age-related macular degeneration and disease burden projection for 2020 and 2040: a systematic review and meta-analysis. Lancet Glob Health. 2014;2:106-16.

3. Sunness JS. The natural history of geographic atrophy, the advanced atrophic form of age-related macular degeneration. Mol Vis. 1999;5:25.

4. Friedman DS, O'Colmain BJ, Munoz B, Tomany SC, McCarty C, De Jong P, Nemesure B, Mitchell P, Kempen J, et al. Prevalence of age-related macular degeneration in the united states. Arch Ophthalmol. 2004;122: 564-72.

5. Holz FG, Schmitz-Valckenberg S, Fleckenstein M. Recent developments in the treatment of age-related macular degeneration. J Clin Investig. 2014;124:1430-8

6. Fleckenstein M, Mitchell P, Freund KB, Sadda S, Holz FG, Brittain C, Henry EC, Ferrara D. The progression of geographic atrophy secondary to age-related macular degeneration. Ophthalmology. 2018;125:369-90.

7. Sunness JS, Gonzalez-Baron J, Applegate CA, Bressler NM, Tian Y, Hawkins B, Barron Y, Bergman A. Enlargement of atrophy and visual acuity loss in the geographic atrophy form of age-related macular degeneration. Ophthalmology. 1999;106:1768-79.

8. Holz FG, Sadda SR, Staurenghi G, Lindner M, Bird AC, Blodi BA, Bottoni F, Chakravarthy U, Chew EY, Csaky K, et al. Imaging protocols in clinical studies in advanced age-related macular degeneration: recommendations from classification of atrophy consensus meetings. Ophthalmology. 2017; 124:464-78

9. Arslan J, Samarasinghe G, Benke KK, Sowmya A, Wu Z, Guymer RH, Baird PN. Artificial intelligence algorithms for analysis of geographic atrophy: A review and evaluation. TransI Vis Sci Technol. 2020;9(2):57.

10. Holekamp N, Wykoff CC, Schmitz-Valckenberg S, Monés J, Souied EH, Lin H, Rabena MD, Cantrell RA, Henry EC, Tang F, et al. Natural history of geographic atrophy secondary to age-related macular degeneration: results from the prospective proxima a and b clinical trials. Ophthalmology. 2020;127(6):769-83.

11. Keenan TD, Agrón E, Domalpally A, Clemons TE, van Asten F, Wong WT, Danis RG, Sadda S, Rosenfeld PJ, Klein ML, et al. Progression of geographic atrophy in age-related macular degeneration: Areds2 report number 16. Ophthalmology. 2018;125:1913-28.

12. Schmitz-Valckenberg S, Sahel J-A, Danis R, Fleckenstein M, Jaffe GJ, Wolf S, Pruente C, Holz FG. Natural history of geographic atrophy progression secondary to age-related macular degeneration (geographic atrophy progression study). Ophthalmology. 2016;123:361-8.

13. Dreyhaupt J, Mansmann U, Pritsch M, Dolar-Szczasny J, Bindewald A Holz F. Modelling the natural history of geographic atrophy in patients with age-related macular degeneration. Ophthalmic Epidemiol. 2005;12: 353-62.

14. Holz FG, Bindewald-Wittich A, Fleckenstein M, Dreyhaupt J, Scholl HP Schmitz-Valckenberg S, Group F-S, et al. Progression of geographic atrophy and impact of fundus autofluorescence patterns in age-related macular degeneration. Am J Ophthalmology. 2007;143:463-72.

15. Feuer WJ, Yehoshua Z, Gregori G, Penha FM, Chew EY, Ferris FL, Clemons TE, Lindblad AS, Rosenfeld PJ. Square root transformation of geographic atrophy area measurements to eliminate dependence of growth rates on baseline lesion measurements: a reanalysis of age-related eye disease study report no. 26. JAMA Ophthalmology. 2013;131:110-1.

16. Box GE, Cox DR. An analysis of transformations. J R Stat Soc Ser B (Methodol). 1964;26:211-43 
17. Lindner M, Bezatis A, Czauderna J, Becker E, Brinkmann CK, Schmitz-Valckenberg S, Fimmers R, Holz FG, Fleckenstein M. Choroidal thickness in geographic atrophy secondary to age-related macular degeneration. Invest Ophthalmol Vis Sci. 2015;56:875-82.

18. Shen L, Liu F, Nardini HG, Del Priore LV. Natural history of geographic atrophy in untreated eyes with nonexudative age-related macular degeneration: a systematic review and meta-analysis. Ophthalmol Retin 2018;2:914-21.

19. Akaike H. A new look at the statistical model identification. IEEE Trans Autom Control. 1974:19(6):716-23.

20. Bates D, Mächler M, Bolker B, Walker S. Fitting linear mixed-effects models using Ime4. J Stat Softw. 2015;67:1-48. https://doi.org/10.18637/ jss.v067.i01.

21. Almohaimeed A, Einbeck J, Almohaimeed MA. Package Boxcoxmix. 2018. R package version 0.21. https://CRAN.R-project.org/package= boxcoxmix. Last accessed: 08 Feb 2021.

22. Sadda SR, Guymer R, Holz FG, Schmitz-Valckenberg S, Curcio CA, Bird AC, Blodi BA, Bottoni F, Chakravarthy U, Chew EY, et al. Consensus definition for atrophy associated with age-related macular degeneration on oct: classification of atrophy report 3. Ophthalmology. 2018;125(4): 537-48.

23. Godunova EK. Jensen inequality. Encyclopedia of Mathematics: Springer Verlag GmbH, European Mathematical Society; 2011. http:// encyclopediaofmath.org/index.php?title=Jensen_inequality\&oldid= 47465. Accessed 08 Feb 2020.

24. van Buuren S, Groothuis-Oudshoorn K. mice: Multivariate imputation by chained equations in r. J Stat Softw. 2011:45:1-67.

25. Kuznetsova A, Brockhoff PB, Christensen RHB. ImerTest package: Tests in linear mixed effects models. J Stat Softw. 2017;82(13):1-26. https://doi. org/10.18637/jss.v082.113.

26. Li JQ, Welchowski T, Schmid M, Mauschitz MM, Holz FG, Finger RP. Prevalence and incidence of age-related macular degeneration in europe: a systematic review and meta-analysis. $\mathrm{Br} J$ Ophthalmol. 2020;104(8):1077-84.

27. Verbeke G, Molenberghs G. Linear Mixed Models for Longitudinal Data. Berlin Heidelberg: Springer Science \& Business Media; 2009.

28. Adrion C, Fleckenstein M, Schmitz-Valckenberg S, Holz F, Mansmann U. Estimation of disease onset for patients with geographic atrophy due to age-related macular degeneration. Gesundheitswesen. 2010;72:207.

29. Heesterbeek TJ, Lorés-Motta L, Hoyng CB, Lechanteur YT, den Hollander Al. Risk factors for progression of age-related macular degeneration. Ophthalmic Physiol Opt. 2020;40(2):140-70.

30. Metelitsina T, Grunwald J, DuPont J, Ying G. Effect of systemic hypertension on foveolar choroidal blood flow in age related macular degeneration. Br J Ophthalmol. 2006;90(3):342-6.

31. Müller PL, Pfau M, Schmitz-Valckenberg S, Fleckenstein M, Holz FG. Optical Coherence Tomography-Angiography in Geographic Atrophy. Ophthalmologica. 2020;244(1):42-50. https://doi.org/10.1159/000510727.

32. Tomany $S$, Wang JJ, Van Leeuwen $R$, Klein $R$, Mitchell $P$, Vingerling JR, Klein BE, Smith W, De Jong PT. Risk factors for incident age-related macular degeneration: pooled findings from 3 continents. Ophthalmology. 2004;111(7):1280-7. https://doi.org/10.1016/j.ophtha 2003.11.010.

33. Buch H, Vinding T, La Cour M, Jensen GB, Prause JU, Nielsen NV. Risk factors for age-related maculopathy in a 14-year follow-up study: the copenhagen city eye study. Acta Ophthalmol Scand. 2005;83(4):409-18.

34. Wu Z, Luu CD, Hodgson LA, Caruso E, Tindill N, Aung KZ, McGuinness MB, Makeyeva G, Chen FK, Chakravarthy U, et al. Prospective longitudinal evaluation of nascent geographic atrophy in age-related macular degeneration. Ophthalmol Retin. 2020;4(6):568-75.

35. Ying G-S, Maguire MG. Complications of Age-related Macular Degeneration Prevention Trial Research Group. Development of a risk score for geographic atrophy in complications of the age-related macular degeneration prevention trial. Ophthalmology. 2011;118(2):332-8. https://doi.org/10.1016/j.ophtha.2010.06.030.

36. Pfau $M$, von der Emde $L$, de Sisternes $L$, Hallak JA, Leng $T$, Schmitz-Valckenberg S, Holz FG, Fleckenstein M, Rubin DL. Progression of photoreceptor degeneration in geographic atrophy secondary to age-related macular degeneration. JAMA Ophthalmol. 2020;138(10): 1026-34.

37. Finger RP, Schmitz-Valckenberg S, Schmid M, Rubin GS, Dunbar $H$, Tufail A, Crabb DP, Binns A, Sánchez Cl, Margaron P, et al. Macustar: development and clinical validation of functional, structural, and patient-reported endpoints in intermediate age-related macular degeneration. Ophthalmologica. 2019;241(2):61-72.

\section{Publisher's Note}

Springer Nature remains neutral with regard to jurisdictional claims in published maps and institutional affiliations.
Ready to submit your research? Choose BMC and benefit from:

- fast, convenient online submission

- thorough peer review by experienced researchers in your field

- rapid publication on acceptance

- support for research data, including large and complex data types

- gold Open Access which fosters wider collaboration and increased citations

- maximum visibility for your research: over $100 \mathrm{M}$ website views per year

At $\mathrm{BMC}$, research is always in progress.

Learn more biomedcentral.com/submissions 